\title{
Optical properties of $\mathrm{ZnO} / \mathrm{ZnS}$ and $\mathrm{ZnO} / \mathrm{ZnTe}$ heterostructures for photovoltaic applications
}

\author{
Joshua Schrier* and Denis O. Demchenko and Lin-Wang Wang \\ Computational Research Division, Lawrence Berkeley National Laboratory, Berkeley, California 94720, USA
}

A. Paul Alivisatos

Materials Science Division, Lawrence Berkeley National Laboratory, Berkeley, California 94720, USA, and Department of Chemistry, University of California, Berkeley, CA 94720, USA.

\begin{abstract}
Although $\mathrm{ZnO}$ and $\mathrm{ZnS}$ are abundant, stable, environmentally benign, their band gap energies $(3.44 \mathrm{eV}, 3.72 \mathrm{eV})$ are too large for optimal photovoltaic efficiency. By using band-corrected pseudopotential density-functional theory calculations, we study how the band gap, optical absorption, and carrier localization can be controlled by forming quantum-well like and nanowire-based heterostructures of $\mathrm{ZnO} / \mathrm{ZnS}$ and $\mathrm{ZnO} / \mathrm{ZnTe}$. In the case of $\mathrm{ZnO} / \mathrm{ZnS}$ core/shell nanowires, which can be synthesized using existing methods, we obtain a band gap of $2.07 \mathrm{eV}$, which corresponds to a Shockley-Quiesser efficiency limit of $23 \%$. Based on these nanowire results, we propose that $\mathrm{ZnO} / \mathrm{ZnS}$ core/shell nanowires can be used as photovoltaic devices with organic polymer semiconductors as p-channel contacts.
\end{abstract}

\section{INTRODUCTION}

The growing awareness of the consequences of greenhouse gas emissions associated with energy production has increased efforts to reduce the cost of solar energy conversion. Economical production of silicon-based photovoltaics are limited by the high cost and energy input required to create the highly purified silicon (comprising 40-50\% of the cost of a finished photovoltaic module). Alternative approaches, such as thin-film devices, rely on toxic and somewhat less abundant CdTe and CdSe, and organic photovoltaics suffer from low efficiency and short-operating lifetimes (due to oxidative damage to the polymers), respectively. ${ }^{1}$ Given this motivation, oxide-based semiconductor materials are attractive due to their abundance, chemical stability, and lack of toxicity-e.g., ZnO is widely used as a paint pigment and as a sunscreen. Besides merely using the bulk, one may also consider whether quantumsize effects may be useful, especially since there is a great deal of expertise in synthesizing ZnO nanowires both by 
vacuum $^{2,3}$ and solution-based ${ }^{4-6}$ methods. In addition, more complicated structures such as nanobelts ${ }^{7,8}$, nanohelices $^{9}$, and others ${ }^{10-12}$ have been synthesized. Unfortunately, the $\mathrm{ZnO}$ band gap (3.4 eV) is too large for use in efficient photovoltaic devices. ${ }^{13}$ Nonetheless, this has not prevented numerous attempts to construct photovoltaics from this material, with schemes such as n- $\mathrm{ZnO} / \mathrm{p}-\mathrm{CdTe}$ thin film heterojunctions, ${ }^{14,15} \mathrm{ZnO} / \mathrm{CdSe}$ composites, ${ }^{16}$ n- $\mathrm{ZnO} / \mathrm{p}-\mathrm{Cu}{ }_{2} \mathrm{O}$ heterojunctions, ${ }^{17,18} \mathrm{n}-\mathrm{ZnO} / \mathrm{p}$-Si heterostructures, ${ }^{19,20} \mathrm{ZnO}$-nanocrystal/organic-polymer hybrid photovoltaics, ${ }^{21-28}$ and $\mathrm{ZnO}$-nanocolumns as electrodes for dye-sensitized photoelectrochemical cells. ${ }^{29}$

One idea to reduce the band gap of $\mathrm{ZnO}$ is to stack it with another environmentally benign and abundant material, such as ZnS. Due to the staggered type-II band alignment, the band gap of the composite structure could be much smaller than either of the individual materials. The oscillator strength for such a transition would be small for two semi-bulk slabs, but in nanometer-scaled structures the spatial proximity of the band-edge wavefunctions to the interface would lead to an increase in the oscillator strength. Thus, it is critically important to know how small a band gap can be in these structures and how large the absorption can be near the band gap. We were encouraged by the variety of efficient experimental methods for the partial conversion of $\mathrm{ZnO}$ nanowires into $\mathrm{ZnO} / \mathrm{ZnS}$ core/shell nanowires. ${ }^{30-36}$

In this letter, we apply theoretical calculations to determine the optical properties of $\mathrm{ZnO} / \mathrm{ZnS}$ and $\mathrm{ZnO} / \mathrm{ZnTe}$ structures, and thus study whether these materials may be practical for photovoltaic devices. Since density functional theory (DFT) calculations perform poorly on the value of the band-gap, we utilize a band-corrected pseudopotential scheme which adds a small empirical correction to the ab initio pseudopotential to reproduce the experimental bulk band-gaps. We then apply this approach to the study of two prototype systems: planar superlattices (shown in Figure 2a) and core/shell quantum wires (shown in Figure 4a), considering $\mathrm{ZnO} / \mathrm{ZnS}$ and ZnO/ZnTe structures. Finally, we discuss the implications of these results for the creation of photovoltaic devices.

\section{METHODS}

Unless otherwise noted, all electronic structure calculations were performed using plane-wave norm-conserving pseudopotential DFT with the PETot code. ${ }^{37}$ In all of the results described here, the $\mathrm{Zn} 3 d$ electrons are included in the valence, and a 75 Ryd kinetic energy cutoff is used for the plane-wave basis. We first obtain the atomic norm-conserving local pseudopotential $V_{l o c}(\vec{r})$ and $\left(s, p, d\right.$-dependent) nonlocal pseudopotential $V_{n o n l o c}(\vec{r})$ from selfconsistent local-density-approximation (LDA) calculations of atoms. However, as a result of the well-known deficiency of LDA, the resulting bulk calculations yield erroneous band gaps of 1.025, 1.981, and $1.163 \mathrm{eV}$ for $\mathrm{ZnO}, \mathrm{ZnS}$, and 
ZnTe, respectively, which are much smaller than the experimental values of $3.44,3.8$, and $2.391 \mathrm{eV} .{ }^{38}$

To correct the band gap error, we modify the nonlocal part of the atomic pseudopotentials for the Zn, O, S, and Te atoms by adding extra potentials $\beta \sin \left(r \pi / r_{c}\right) / r$ (zero outside $r_{c}$, where $r_{c}$ is the cutoff radius used for the pseudopotential) to each of the $s, p, d$ nonlocal parts, where $\beta$ are empirical parameters fitted to reproduce the experimental bulk band gaps and other high-symmetry $k$-point band energies, ${ }^{38-41}$ as shown in Supplementary Table IS; we also fix the energy of the valence band maximum (VBM) to its original LDA result to avoid altering the valence band alignment. This "band-corrected pseudopotential" approach has been used previously for the treatment of nanocrystals ${ }^{42}$ and isovalent impurities ${ }^{43}$. To minimize concerns about transferability, we fitted separate ZnO/ZnS and $\mathrm{ZnO} / \mathrm{ZnTe}$ sets of parameters $\beta$, shown in Supplementary Tables IIS and IIIS, respectively. In the case of ZnTe we have also included the spin-orbit coupling using the relativistic Te pseudopotential yielding the experimental valence band splitting of $0.91 \mathrm{eV} .{ }^{38}$ Following this procedure, we obtain band gaps of $3.334 / 3.716$ for the $\mathrm{ZnO} / \mathrm{ZnS}$ fitting, and 3.361/2.337 for the $\mathrm{ZnO} / \mathrm{ZnTe}$ fitting, with good $(\sim 0.1 \mathrm{eV})$ agreement with the experimental results.

To summarize, in the electronic structure calculations described below, we first perform a self-consistent calculation using the original $a b$ initio pseudopotentials to yield the local potential, then (non-self-consistently) calculate the corrected eigenenergies with this local potential plus the band-corrected nonlocal pseudopotentials. The wavefunctions and eigenenergies obtained from this step are used for the subsequent absorption spectrum calculations and wavefunction plots. The imaginary part of the dielectric function, which is proportional to the optical absorption spectrum by a volume factor, is calculated as a function of the photon frequency, $\omega, \mathrm{as}^{44}$

$$
\epsilon_{i}(\omega)=\frac{8 \pi^{2} e^{2} \hbar^{2}}{3 m^{2}(\hbar \omega)^{2} V} \sum_{k} \sum_{c, v}\left|\left\langle\phi_{c}(k)|\hat{p}| \phi_{v}(k)\right\rangle\right|^{2} \delta\left(E_{c}(k)-E_{v}(k)-\hbar \omega\right),
$$

where $e, m$, and $V$ are the electron charge, electron mass, and volume of the system, the summations over $k, c$, and $v$ run over the k-points, conduction-bands, and valence bands, respectively, for the eigenstates $\phi_{c}(k)$ and eigenenergies $E_{c}(k)$, respectively, and the $\hat{p}$ is the momentum operator. The spectra results shown in Figure 2 and Figure 4 include a gaussian broadening term of $20 \mathrm{meV}$. 

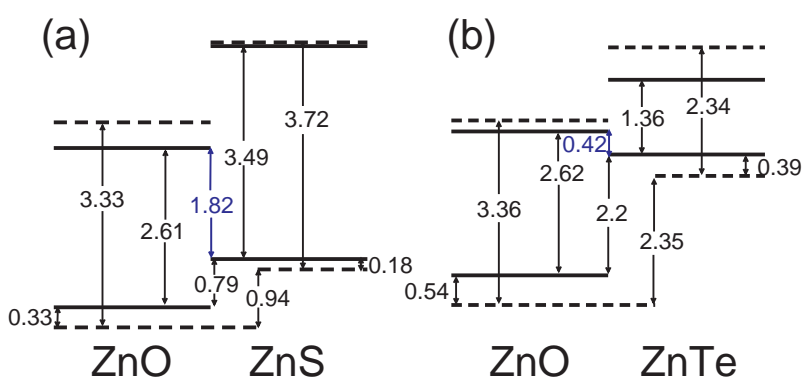

FIG. 1: Calculated VBM and CBM alignments for (a) $\mathrm{ZnO} / \mathrm{ZnS}$ and (b) ZnO/ZnTe. The solid lines show the strained band alignments for the heterostructures similar to Figure 2a, and the dashed lines show the calculated natural band alignments. The blue arrows indicate the effective band gap due to the transition from the VBM of $\mathrm{ZnS}$ (ZnTe) to the CBM of ZnO.

\section{RESULTS}

\section{A. Bulk heterostructures}

In this section, we examine the cases of planar heterostructure superlattices, shown schematically in Figure 2a. To obtain the geometry we constructed a model consisting of the interfaces between zincblende $\mathrm{ZnO}$ and $\mathrm{ZnS}$ (ZnTe) slabs in the (001) direction each consisting of 7 monolayers (Figure 2a). There is significant lattice mismatch, since the corresponding bulk zincblende lattice constants for ZnO, ZnS, and ZnTe are $4.6 \AA$, $5.42 \AA$, and $6.1 \AA$, respectively. To find the optimal geometry within such planar zinc-blende structure, the supercell was optimized with respect to three independent lattice parameters: $a, c_{1}, c_{2}$, where $c_{1}$ and $c_{2}$ are the $z$-direction lattice constants in the $\mathrm{ZnO}$ and $\mathrm{ZnS}(\mathrm{ZnTe})$ slabs in the supercell, respectively, and $a$ is the lattice constant along the $x$ - and $y$-directions. This relaxation was performed by ultrasoft-pseudopotential projector augmented wave (PAW) generalized gradient approximation (GGA) DFT calculations, using the commercial VASP program. ${ }^{45}$ From this geometry, the optical properties were calculated using the norm-conserving pseudopotential/band-correction approach discussed in Section II, using 200 irreducible $k$-points to sample the Brillouin zone.

We first examine the bulk band alignments of $\mathrm{ZnO} / \mathrm{ZnS}$ and $\mathrm{ZnO} / \mathrm{ZnTe}$. Our calculated results are shown in Figure

1. The valence band alignments were determined from the difference of the VBM energies of the bulk materials (calculated by self-consistent LDA with the $a b$ initio pseudopotentials), then adjusted by the offset of the electrostatic potential in the strained superlattice. The natural valence band alignments (defined as the band alignment when the two materials are both at their natural lattice constants without strain) were then deduced by subtracting both the uniaxial and hydrostatic strain effects using the recent absolute valence band deformation potentials of Li et $^{\text {al. }}{ }^{46,47}$ 
The conduction bands were then determined by adding the gaps calculated with the band-corrected pseudopotentials for strained and unstrained bulk materials. In the present calculations, the effect of the crystal-field splitting (due to uniaxial strain) is much larger than the absolute deformation potential term (due to hydrostatic strain), which explains why even though the volume changes have opposite signs for the $\mathrm{ZnO}$ and $\mathrm{ZnS}(\mathrm{ZnTe})$ regions, the strained VBM energies are both higher than the natural band energies for both materials. Our calculated natural valence band alignments are in good agreement with recent theoretical results by Moon et al., ${ }^{48}$ while it is in disagreement with the earlier band alignment results of Ref. 49 for the reasons discussed in Ref. 46 . Note that our subsequent calculations are performed directly, and do not suffer from any of the assumptions of the nature of the absolute deformation potentials. For $\mathrm{ZnO} / \mathrm{ZnS}$ (Figure 1a) we observe a staggered Type-II band alignment for both the strained and natural cases. The strain has a large effect, shifting the band gaps from the unstrained bulk values (dashed lines) of 3.334/3.716 eV for $\mathrm{ZnO} / \mathrm{ZnS}$ to the strained bulk values (solid lines) of 2.614/3.494 eV. Similarly, due to the even larger lattice mismatch between the $\mathrm{ZnO} / \mathrm{ZnTe}$ interface (Figure 1b), the band gaps are shifted from $3.361 / 2.337$ eV for unstrained $\mathrm{ZnO} / \mathrm{ZnTe}$ (dashed lines) to $2.615 / 1.364 \mathrm{eV}$ for the strained bulk materials (solid lines). The strain also reduces the energy of the transition between the top of the bulk ZnS (or ZnTe) valence band to the bottom of the bulk $\mathrm{ZnO}$ conduction band (without the quantum confinement effects discussed below), from $2.39 \mathrm{eV}$ to $1.82 \mathrm{eV}$ in $\mathrm{ZnO} / \mathrm{ZnS}$ and from $1.01 \mathrm{eV}$ to $0.42 \mathrm{eV}$ in $\mathrm{ZnO} / \mathrm{ZnTe}$. Thus, the strain can also be used to reduce the total system band gap. Depending on the geometry of the nanostructure, the strain effect can be varied, providing a degree of freedom for band gap engineering.

To study the effects of nanometer scale confinement in superlattices, we examined first the ZnO/ZnS heterostructure, shown in Figure 2a and Figure 2b, using the band-corrected pseudopotential approach described in Section II. The first absorption peak occurs at $2.316 \mathrm{eV}$ (indicated by the red arrow in Figure 2b). This corresponds to the direct transition between the valence band maximum (VBM) and conduction band minimum (CBM) in the interface area, as can be seen clearly in the VBM and CBM wavefunctions plotted in Figure 3a,b. The band edge states are predominantly localized either in one material or the other, but that the interface atoms have significant overlap of the VBM and CBM. We find that the oscillator strength of lowest energy transition is $3 \%$ of the magnitude of the VBM-to-CBM transition in bulk ZnO. The imaginary part of the dielectric constant at the VBM-to-CBM edge is 0.017, which is quite small. While from the band alignment of Figure 1a, it might be expected that the absorption onset should occur at $1.8 \mathrm{eV}$, in this structure, the quantum confinement effects lead to an increase in the band gaps in the superlattice. This illustrates a trade-off when making these types of nanometer-scale structures: namely, 


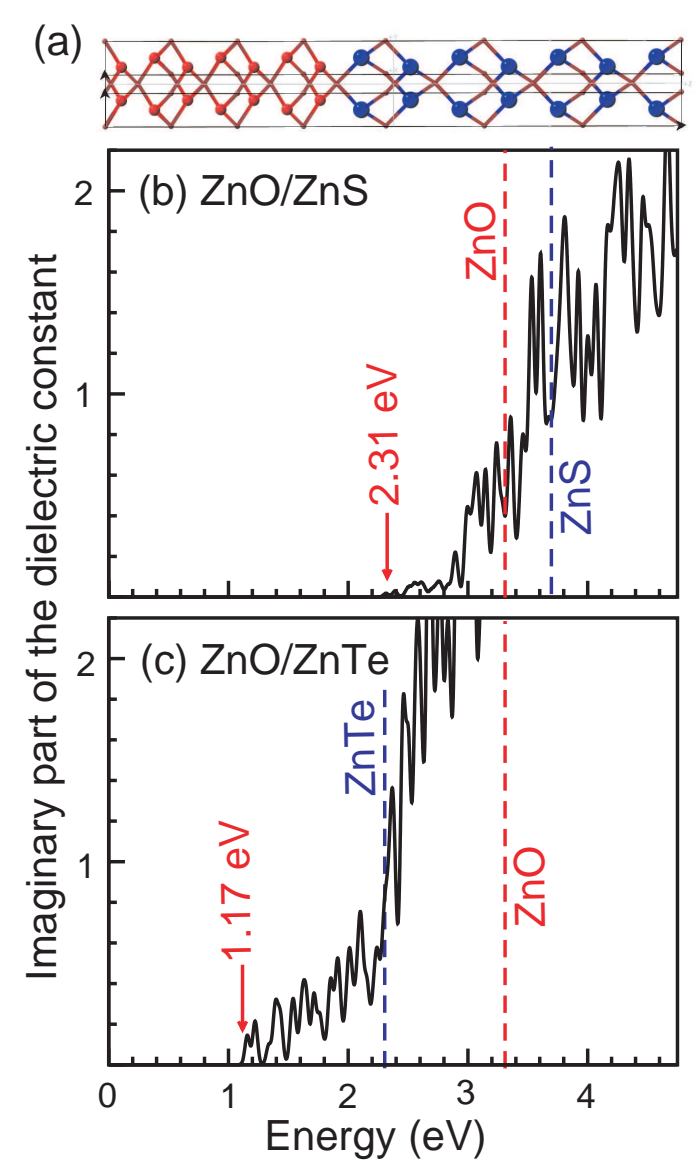

FIG. 2: (a) Relaxed geometry for the $\mathrm{ZnO} / \mathrm{ZnS}$ bulk heterostructure superlattice interface, demonstrating the type of geometry discussed in Section III A. Below are the calculated optical-absorption for the (b) ZnO/ZnS and (c) ZnO/ZnTe bulkheterostructures. The red arrows indicate the values of the lowest observed peaks, and the vertical red $(\mathrm{ZnO})$ and blue $(\mathrm{ZnS}$ or $\mathrm{ZnTe}$ ) dashed lines correspond to the bulk band gaps. The sharp peak features result from the finite (200) number of $k$-points used in the calculation.

decreasing the layer thickness improves the oscillator strength for transitions between the two materials by bringing the spatially-localized band-edge wavefunctions closer to each other, but at the same time, quantum confinement effects increase the band gap which is quite the opposite of our goal.

Next we consider a similar type of planar heterostructure comprised of $\mathrm{ZnO} / \mathrm{ZnTe}$. In Figure 2c, see the first peak at $1.168 \mathrm{eV}$, similar to the bulk $\mathrm{Si}$ band gap. As in the previous $\mathrm{ZnO} / \mathrm{ZnS}$ case, the band-edge wavefunctions for this $\mathrm{ZnO} / \mathrm{ZnTe}$ heterostructure are localized in the two different materials, as shown in Figure 3c,d, and this lowest transition has an oscillator strength which is $4 \%$ of the direct VBM-to-CBM transition in bulk ZnO. The imaginary part of the dielectric constant at $1.2 \mathrm{eV}$ is 0.11 , much greater than silicon at this wavelength. The increase in the dielectric constant, as compared to the $\mathrm{ZnO} / \mathrm{ZnS}$ structure discussed above, results from a combination of the higher 
(a) $\mathrm{ZnO} / \mathrm{ZnS}$ VBM

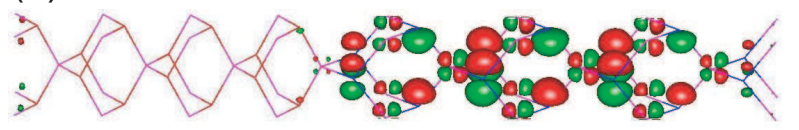

(b) $\mathrm{ZnO} / \mathrm{ZnS} \mathrm{CBM}$

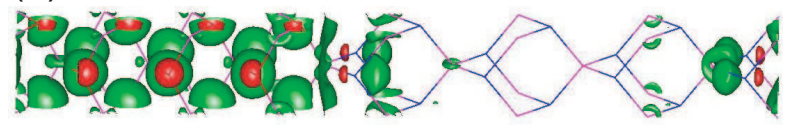

(c) $\mathrm{ZnO} / \mathrm{ZnTe} \mathrm{VBM}$

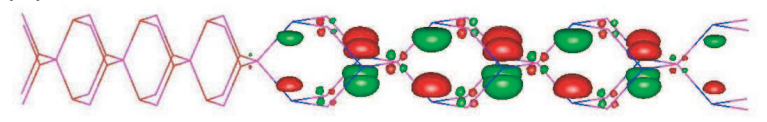

(d) $\mathrm{ZnO} / \mathrm{ZnTe} \mathrm{CBM}$

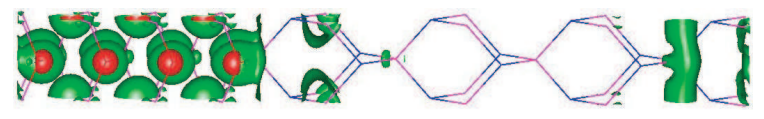

FIG. 3: Band edge wavefunction plots for the bulk heterostructures, with positive and negative signs indicated by green and red, respectively. (a) Valence band maximum (VBM), and (b) conduction band minimum (CBM) for ZnO/ZnS heterostructure. (c) VBM, and (d) CBM for ZnO/ZnTe heterostructure. The ZnO/ZnTe plots, which were calculated including spin-orbit coupling, only show the real part of the spin-up component; the spin-down component is qualitatively similar.

oscillator strength and the lower energy in the denominator term of Eq.(1). As in the case of the $\mathrm{ZnO} / \mathrm{ZnS}$ case, quantum confinement has significantly increased the band gap as compared to the strained band alignment in Figure $1 b$.

\section{B. Heterostructured quantum wires}

For the superlattice heterostructures discussed above, there is a trade off between the oscillator strength and quantum confinement effects for solar cell applications, especially for $\mathrm{ZnO} / \mathrm{ZnS}$. In addition, the large elastic strain might render synthesis of the large area superlattice heterostructures difficult. Thus, it is worthwhile to explore other geometries where the strain profiles are different. We focus here on $\mathrm{ZnO} / \mathrm{ZnS}$, due to its benign environmental properties, and the need to further reduce the band gap identified in the above discussion. Here we examine $\mathrm{ZnO} / \mathrm{ZnS}$ core/shell quantum wire, shown schematically in Figure 4a. This structure consists of an interior core of $\mathrm{ZnO}$ with radius $11.7 \AA$ (about 4 monolayers), surrounded by a shell of 4 monolayers of ZnS, for a total radius of $23.4 \AA$. This relatively small nanowire is considered to obtain appreciable oscillator strength. Similar $\mathrm{ZnO} / \mathrm{ZnS}$ core/shell nanowires have been synthesized experimentally although with much larger diameter. ${ }^{2,4,30-36}$ We note that our structure is much larger than the very narrow $\mathrm{ZnO}$ nanowires which recent DFT calculations have shown to undergo structural 


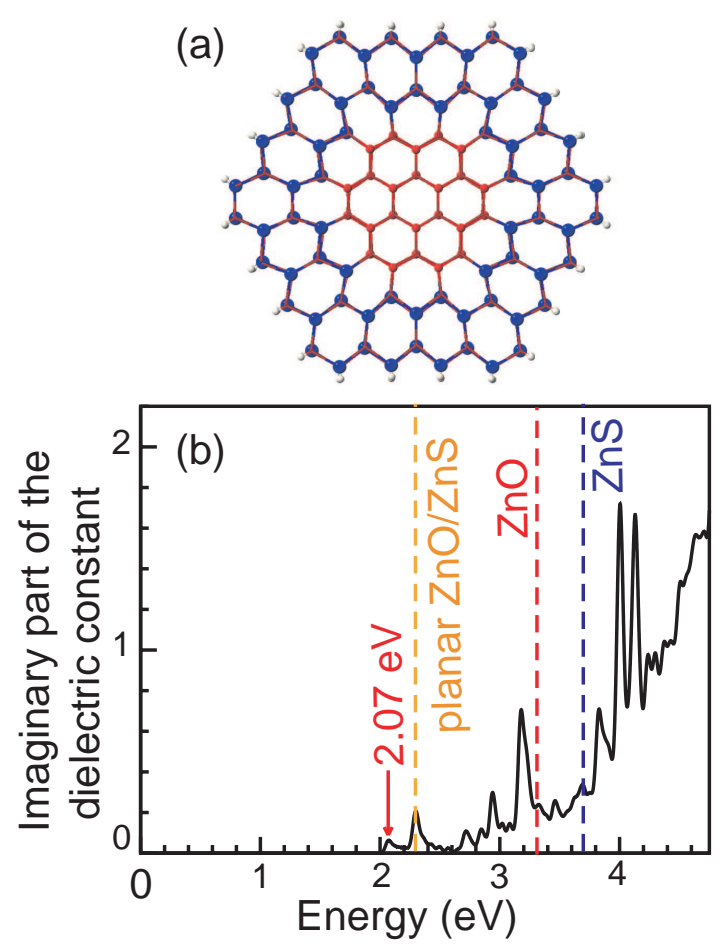

FIG. 4: (a) Relaxed geometry for the $\mathrm{ZnO} / \mathrm{ZnS}$ core/shell nanowire discussed in Section III B. (b) Calculated optical-absorption using the band-corrected pseudopotentials. The vertical red $(\mathrm{ZnO})$ and blue $(\mathrm{ZnS})$ dashed lines correspond to the bulk band gaps, and the vertical orange line corresponds to the band gap of the planar $\mathrm{ZnO} / \mathrm{ZnS}$ heterostructure of Figure $2 \mathrm{~b}$, discussed in Section III A.

transformations to graphitic structures, ${ }^{50}$ so we use the wurtzite structure. Structural relaxation were performed using the generalized valence force field (G-VFF) model. ${ }^{51}$ The parameterizations developed to reproduce the experimentally observed elastic constants (from Ref. 38) are shown in Supplementary Table IVS. In the case of ZnO, we used the relationship of Martin ${ }^{52}$ to transform the experimental wurtzite elastic constants into the equivalent (hypothetical) zinc-blende form used to fit the G-VFF parameters. For the optical calculations 25 irreducible $k$-points are used to sample the periodicity along the wire axis.

Although the first pronounced peak in Figure 4 occurs at $2.070 \mathrm{eV}$, this is due to transitions from the two valence band states below the VBM (shown in Figure 5a,b) to the CBM (Figure 5d); the transition between the VBM (Figure 5c) and CBM at $1.935 \mathrm{eV}$ is zero due to symmetry. This is due to an inplane anti-inversion symmetry of the VBM envelope function, as shown in Figure 5c, resulting in an envelope-function induced dark exciton. For solar cell applications, this dark exciton can suppress electron-hole recombination, thus enhancing the carrier collection efficiency. 

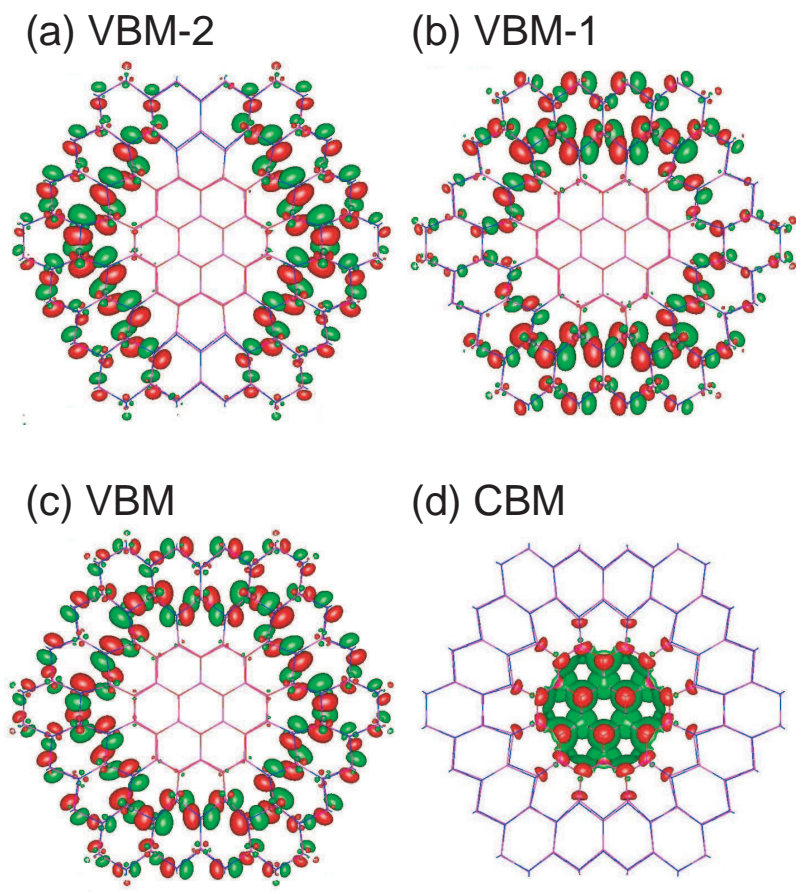

(d) CBM

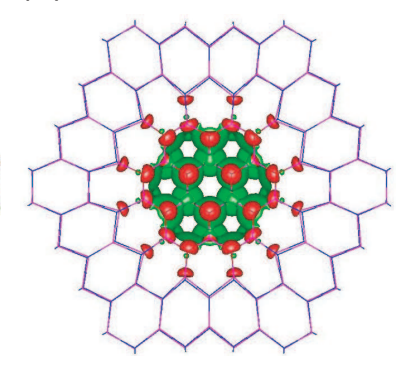

FIG. 5: Band edge wavefunctions of the $\mathrm{ZnO} / \mathrm{ZnS}$ core/shell nanowire calculated in Section III B, with positive and negative signs indicated by green and red, respectively. (a) VBM-2 ; (b) VBM-1; (c) VBM; (d) CBM. The peak at 2.07 eV in Figure 4 arise from the transition between $(\mathrm{a}) \rightarrow(\mathrm{d})$ and $(\mathrm{b}) \rightarrow(\mathrm{d}) ;(\mathrm{c}) \rightarrow(\mathrm{d})$ has a very small oscillator strength.

The absorption peak at $2.070 \mathrm{eV}$ is a $0.3 \mathrm{eV}$ improvement upon the $\mathrm{ZnO} / \mathrm{ZnS}$ heterostructure case studied in Section III A, and the imaginary dielectric constant at this peak is 0.06 , which is also larger than that of the first peak in the $\mathrm{ZnO} / \mathrm{ZnS}$ superlattice (Figure $2 \mathrm{~b}$ ). Thus, the $\mathrm{ZnO} / \mathrm{ZnS}$ core/shell nanowire improves upon both the bandgap and oscillator strength of its superlattice counterpart. However, the core/shell nanowire does have reduced absorption at higher photon energies when compared to the planar $\mathrm{ZnO} / \mathrm{ZnS}$ heterostructure (Figure $2 \mathrm{~b}$ ), although this is not too grave of a problem for solar cell applications, since it is still larger than the lower energy absorption. The valence bandedge wavefunctions responsible for the first absorption peak (Figure 5a-b) are predominantly localized in the exterior $\mathrm{ZnS}$ layer and the CBM (Figure 5d) predominantly localized in the the interior $\mathrm{ZnO}$ core, much like the behavior for the planar heterostructure in Figure 3a,b. From our analysis in the previous section on the competition between strain and quantum confinement effects, we can make a few general remarks on the properties of larger structures. First, strain is a useful property to take advantage of as it is observed to reduce the band gap for these materials, and these types of nanostructures can accommodate strains which may not be possible in planar heterostructures. Second, as the size of the system is increased, quantum confinement effects would be reduced, which leads to a smaller band gap, although at the penalty of a smaller oscillator strength. To estimate how large an oscillator strength is sufficient for 


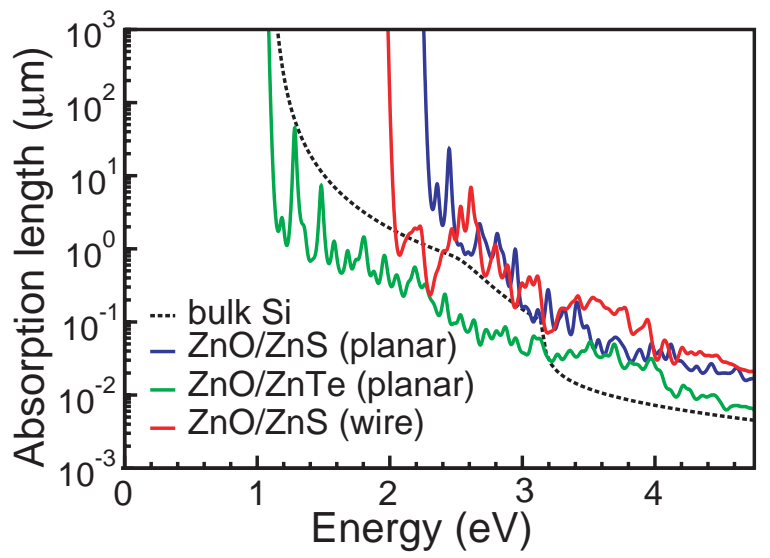

FIG. 6: Absorption length calculations, as a function of light energy. The blue and green lines indicate the values calculated for the $\mathrm{ZnO} / \mathrm{ZnS}$ and $\mathrm{ZnO} / \mathrm{ZnTe}$ planar heterostructures discussed in Section III A; the red line indicates the value calculated for the $\mathrm{ZnO} / \mathrm{ZnS}$ core/shell nanowire discussed in Section III B. The dotted black line shows the experimental data for bulk Si at $300 \mathrm{~K} .^{53-55}$

photovoltaic applications, one can compare the absorption coefficient near the band edge to that of bulk $\mathrm{Si}$ at room temperature. ${ }^{53}$ The bulk Si near edge absorption comes from the phonon-assisted transition, which is much smaller than the band edge absorption of 0.06 calculated for the $\mathrm{ZnO} / \mathrm{ZnS}$ nanowire. To emphasize this point, in Figure 6 we show the calculated absorption length (in microns) for the planar and nanowire nanostructures studied in the present paper, as compared to the experimental data for bulk silicon at $300 \mathrm{~K} \cdot{ }^{53-55}$ We find that all of the nanostructures studied in this work have an absorption length on the order of microns, comparable to that of bulk silicon. Thus an equivalent overall volume of the packed nanowires will be sufficient to absorb incident light as well as Si devices.

Photovoltaic device operation involves three processes: light absorption, carrier separation, and carrier collection. Concerning light absorption, as shown in Figure 6, a similar film thickness is required as in bulk Si solar cells. This can be achieved by growing long $\mathrm{ZnO}$ nanowires ${ }^{2,4,6}$ and packing many nanowires together on a surface. Concerning carrier separation, the natural separation of the electron and hole into the $\mathrm{ZnO}$ and $\mathrm{ZnS}$ regions reduces the barrier to exciton dissociation and carrier separation under the electric field of the electrodes. Finally, concerning carrier collection in the contacts, we can take advantage of the localization of the VBM in the exterior ZnS layer (as shown in Figure 5a) for these core/shell wires. One can imagine constructing a device in which the ZnO makes a direct connection to one contact (e.g., a conductive glass substrate ${ }^{58}$ ), and the hole, after separating into the ZnS region is extracted from the nanowire into a surrounding p-channel organic semiconductor, e.g. poly(3-hexylthiophene) 
(P3HT), which are typically more conductive and air stable than typical $n$-channel polymers. ${ }^{59}$

\section{CONCLUSIONS}

The calculations presented in this work demonstrate that the formation of $\mathrm{ZnO} / \mathrm{ZnS}$ and $\mathrm{ZnO} / \mathrm{ZnTe}$ nanoheterostructures can substantially reduce the optical band-gap, while simultaneously maintaining required optical absorption. Using the Shockley-Quiesser ${ }^{13}$ model as guidance for the theoretical limit of ideal solar cell efficiency, we obtain efficiency limits of $19 \%$ and $30 \%$ for planar $\mathrm{ZnO} / \mathrm{ZnS}$ and $\mathrm{ZnO} / \mathrm{ZnTe}$ quantum-well heterostructures and $23 \%$ for $\mathrm{ZnO} / \mathrm{ZnS}$ core/shell quantum wires. In all cases, this shows a dramatic improvement over bulk $\mathrm{ZnO}$, which has a theoretical efficiency limit of only $7 \%$. We also find dark exciton formation in the ZnO/ZnS nanowire which could improve the carrier collection in solar cells by reducing the exciton recombination rate. In conclusion, the current results demonstrate the feasibility of the scheme proposed in Section III B, and it is our hope that this will stimulated more detailed experimental investigation. Many other features of the $\mathrm{ZnO} / \mathrm{ZnS}$ system can be explored in the future to further reduce the band gap and increase the oscillator strength, including piezoelectric effects, alloying, and interfacial roughening.

\section{Acknowledgments}

This work was supported by the U. S. Department of Energy under Contracts No. DE-AC03-76SF00098 and DE-AC02-05CH11231, and used the resources of the National Energy Research Scientific Computing Center.

Supporting Information Available: Tables listing the band-structure fitting points, band-correction potential terms, and the bulk elastic constants. This material is available free of charge via the Internet at http://pubs.acs.org

* Electronic address: jschrier@lbl.gov

1 U.S. Department of Energy; Basic Research Needs for Solar Energy Utilization, 2005.

${ }^{2}$ Goldberger, J.; Sirbuly, D. J.; Law, M.; Yang, P. J. Phys. Chem. B 2005, 109, 9.

${ }^{3}$ Chang, P.-C.; Chien, C.-J.; Stichtenoth, D.; Ronning, C.; Lu, J. G. Appl. Phys. Lett. 2007, 90, 113101.

${ }^{4}$ Green, L. E.; Yuhas, B. D.; Law, M.; Zitoun, D.; Yang, P. Inorg. Chem. 2006, 45, 7535.

5 Anthony, S. P.; Lee, J. I.; Kim, J. K. Appl. Phys. Lett. 2007, 90, 103107.

${ }^{6}$ Liu, J.; Huang, X.; Li, Y.; Ji, X.; Li, Z.; He, X.; Sun, F. J. Phys. Chem. C 2007, 111, 4990. 
7 Pan, Z. W.; Dai, Z. R.; Wang, Z. L. Science 2001, 291, 1947.

${ }^{8}$ Yang, J.; Liu, G.; Lu, J.; Qiu, Y.; Yang, S. Appl. Phys. Lett. 2007, 90, 103109.

${ }^{9}$ Gao, P. X.; Ding, Y.; Mai, W.; Hughes, W. L.; Lao, C.; Wang, Z. L. Science 2005, 309, 1700.

${ }^{10}$ Wang, Z. L.; Kong, X. Y.; Ding, Y.; Gao, P.; Hughes, W. L.; Yang, R.; Zhang, Y. Adv. Funct. Mater. 2004, $14,944$.

11 Wang, X.; Song, J.; Wang, Z. L. Chem. Phys. Lett. 2006, 424, 86.

12 Cao, B.; Teng, X.; Heo, S. H.; Li, Y.; Cho, S. O.; Li, G.; Ci, W. J. Phys. Chem. C 2007, 111, 2470.

13 Shockley, W.; Queisser, H. J. Appl. Phys. Lett. 1961, 32, 510.

14 Tomar, M. S. Thin Solid Films 1988, 164, 295.

15 Wary, G.; Kachary, T.; Rahman, A. Int. J. Thermophys. 2006, 27, 332.

${ }^{16}$ Gomez-Daza, O.; Campos, J.; Sanchez, A.; Banos, L.; Nair, M. T. S.; Nair, P. K. J. Electrochem. Soc. 2001, 148 , G330.

17 Izaki, M.; Mizuno, K.; Shinagaw, T.; Inaba, M.; Tasaka, A. J. Electrochem. Soc. 2006, 153 , C668.

18 Minami, T.; Miyata, T.; Ihara, K.; Minamino, Y.; Tsukada, S. Thin Solid Films 2006, 494, 47.

19 Fu, Z. X.; Lin, B. X.; Liao, G. H. Chin. Phys. Lett. 1999, 16, 753.

${ }^{20}$ Liu, C. H.; Chen, Y. L.; Lin, B. X.; Zhu, J. J.; Fu, Z. X.; Peng, C.; Yang, Z. Chin. Phys. Lett. $2001,18,1108$.

${ }^{21}$ Beek, W. J. E.; Wienk, M. M.; Janssen, R. A. J. Adv. Mater. 2004, 16, 1009.

22 Beek, W. J. E.; Wienk, M. M.; Janssen, E. A. J. J. Mater. Chem. 2005, 15, 2985.

${ }^{23}$ Beek, W. J. E.; Wienk, M. M.; Kemerink, M.; Yang, X.; Janssen, R. A. J. J. Phys. Chem. B 2005, $109,9505$.

${ }^{24}$ Beek, W. J. E.; Sloof, L. H.; Wienk, M. M.; Kroon, J. M.; Janssen, R. A. J. Adv. Funct. Mater. $2005,15,1703$.

${ }^{25}$ Beek, W. J. E.; Wienk, M. M.; Janssen, R. A. J. Adv. Funct. Mater. 2006, 16, 1112.

${ }^{26}$ Peir'o, A. M.; Ravirajan, P.; Govender, K.; Boyle, D. S.; OBrien, P.; Bradley, D. D. C.; Nelson, J.; Durrant, J. R. J. Mater. Chem. 2006, 16, 2088.

27 Olson, D. C.; Piris, J.; Collins, R. T.; Shaheen, S. E.; Ginley, D. S. Thin Solid Films 2006, $496,26$.

${ }^{28}$ Lira-Cantu, M.; Krebs, F. C. Sol. Energy Mater. Sol. Cells 2006, 90, 2076.

${ }^{29}$ Keis, K.; Bauer, C.; Boschloo, G.; Hagfeldt, A.; Westermark, K.; Rensmo, H.; Siegbahn, H. J. Photochem. Photobio. A: Chem. 2002, 148, 57.

${ }^{30}$ Dloczik, L.; Engelhardt, R.; Ernst, K.; Fiechter, S.; Sieber, I.; Könenkamp, R. Appl. Phys. Lett. $2001,78,3687$.

31 Lokhande, C.; Pathan, H. M.; Giersig, M.; Tributsch, H. Appl. Surf. Sci. 2002, $187,101$.

32 Könenkamp, R.; Dloczik, L.; Ernst, K.; Olesch, C. Physica E 2002, 14, 219.

33 Sankapal, B. R.; Sartale, S. D.; Lokhande, C. D.; Ennaoui, A. Sol. Energy Mater. Sol. Cells 2004, 83, 447.

34 Zhang, H.; Yang, D.; Ma, X.; Que, D. Nanotechnology 2005, 16, 2721.

35 Yan, C.; Xue, D. J. Phys. Chem. B 2006, 110, 25850.

36 Panda, S. K.; Dev, A.; Chaudhuri, S. J. Phys. Chem. C 2007, 111, 5039. 
37 http://hpcrd.lbl.gov/ linwang/petot/petot.html.

38 Rössler, U., Ed. Semiconductors, II-VI and I-VII Compounds: Semimagnetic Compounds, Vol. 41B of Landolt-Börnstein, New Series, Group III; Springer: Heidelberg, 1999.

39 Martins, J.; Troullier, N.; Wei, S.-H. Phys. Rev. B 1991, 43, 2213.

${ }^{40}$ Walter, J. P.; Cohen, M. L.; Petroff, Y.; Balkanski, M. Phys. Rev. B 1970, 1, 2661.

41 Usuda, M.; Hamada, N.; Kotani, T.; van Schilfgaarde, M. Phys. Rev. B 2002, 66, 125101.

${ }^{42}$ Li, J.; Wang, L.-W. Phys. Rev. B 2005, 72, 125325.

43 Li, J.; Wei, S.-H. Phys. Rev. B 2006, 73, 041201.

44 Wang, L.-W. Phys. Rev. B 1994, 49, 10154.

${ }^{45}$ Kresse, G.; Furthmüller, J. Comput. Mater. Sci. 1996, 6, 15.

${ }^{46}$ Li, Y.-H.; Gong, X. G.; Wei, S.-H. Appl. Phys. Lett. 2006, 88, 042104.

${ }^{47}$ Li, Y.-H.; Gong, X. G.; Wei, S.-H. Phys. Rev. B 2006, 73, 245206.

${ }^{48}$ Moon, C.-Y.; Wei, S.-H.; Zhu, Y. Z.; Chen, G. D. Phys. Rev. B 2006, 74, 233202.

49 Wang, L.; unger, A. J. Phys. Chem. B 1998, 102, 6449.

50 Zhang, L.; Huang, H. Appl. Phys. Lett. 2007, 90, 023115.

${ }^{51}$ Williamson, A. J.; Wang, L. W.; Zunger, A. Phys. Rev. B 2000, 62, 12963.

${ }^{52}$ Martin, R. M. Phys. Rev. B 1972, 6, 4546.

53 Jellison, G. E.; Modine, F. A. Phys. Rev. B 1983, 27, 7466.

54 Rajkanan, K.; Singh, R.; Shewchun, J. Solid-State Electronics 1979, 22, 793.

${ }^{55}$ Groom, D. E.; Holland, S. E.; Levi, M. E.; Palaio, N. P.; Perlmutter, S.; Stover, R. J.; Wei, M. SPIE 1999, $3649,90$.

${ }^{56}$ Gregg, B. A.; Hanna, M. C. J. Appl. Phys. 2003, 93, 3605.

57 Kayes, B. M.; Atwater, H. A.; Lewis, N. S. J. Appl. Phys. 2005, 97, 114302.

${ }^{58}$ Xu, L.; Liao, Q.; Zhang, J.; Ai, X.; Xu, D. J. Phys. Chem. C 2007, 111, 4549.

${ }^{59}$ Newman, C. R.; Frisbie, C. D.; da Silva Filho, D. A.; Brédas, J.-L.; Ewbank, P. C.; Mann, K. R. Chem. Mater. 2004, 16, 4436. 